\title{
ПРОЦЕССЫ УПРАВЛЕНИЯ
}

\author{
УДК 517.977.1
}

A. D. Khamitova

\section{CHARACTERISTIC POLYNOMIALS FOR A CYCLE OF NON-LINEAR DISCRETE SYSTEMS WITH TIME DELAYS}

Odessa National Polytechnic University, 1, Shevchenko Ave, Odessa, 65044, Ukraine

\begin{abstract}
We study a method associated with constructing of delayed feedback for local stabilization of periodic orbits of nonlinear discrete systems. An alternative approach to the construction of characteristic polynomial for the delay system linearized in the neighborhood of $T$-cycle is suggested. It is proven that our new alternative approach is equivalent to the standard one, however, it allows us to produce directly new forms of polynomials. These forms are convenient in applications to the problems of chaos control and allow us to apply methods of geometric complex function theory. This article is an extension of the results, which received D. Dmitrishin, P. Haglstein, A. Khamitova and A. Stokolos to the vector case. Refs 6. Fig 1.
\end{abstract}

Keywords: Non-linear systems, asymptotic stability of cycles, DFC methods.

\section{А. Д. Хамитова}

\section{ХАРАКТЕРИСТИЧЕСКИЕ ПОЛИНОМЫ ЦИКЛОВ НЕЛИНЕЙНЫХ ДИСКРЕТНЫХ СИСТЕМ С ЗАПАЗДЫВАНИЕМ}

Одесский Национальный политехнический университет, Украина, 65044, Одесса, пр. Шевченко, 1

\begin{abstract}
Изучается один из методов локальной стабилизации периодических орбит нелинейных дискретных систем, связанный с построением запаздывающей обратной связи. Предлагается альтернативный подход к построению характеристического полинома для линеаризованной в окрестности $T$-цикла системы с запаздыванием. Доказано, что альтернативный подход эквивалентен стандартным, однако напрямую дает возможность получать новые формы характеристических полиномов. Эти формы оказываются удобными в решении проблемы управления хаосом и позволяют применить методы геометрической теории функции комплексного переменного. В статье приведены и обсуждены результаты, полученные Д. Дмитришиным, П. Хагелстейном, А. Хамитовой и А. Стоколосом на векторный случай. Библиогр. 6 назв. Ил. 1.

Ключевые слова: нелинейные системы, асимптотическая стабильность циклов, ДФК методы.
\end{abstract}

1. Introduction. The goal of the current paper is to introduce a modified approach to the problem of local asymptotic stability of cycles in special non-linear systems and to

Khamitova Anna Dmitrievna - postgraduate student; anna_khamitova@georgiasouthern.edu

Хамитова Анна Дмитриевна - аспирант; anna_khamitova@georgiasouthern.edu

(C) Санкт-Петербургский государственный университет, 2016 
make a comparison. The standard approach to this problem is well outlined in $[1,2]$ which is a further development of the method describe by Ö. Morgül [3].

We will distinguish between the systems with no time delays

$$
x_{k+1}=f\left(x_{k}\right), f \in \mathbb{R}^{m}, k=0,1, \ldots,
$$

and with time delays of the form

$$
x_{k+1}=a_{1} f\left(x_{k}\right)+a_{2} f\left(x_{k-T}\right)+\ldots+a_{N} f\left(x_{k-(N-1) T}\right), f \in \mathbb{R}^{m}, k=0,1, \ldots, T \in \mathbb{Z}_{+} .
$$

Note that the systems (2) appear in the problems of localization of unknown periodic orbits and control of chaos.

In scalar case the standard approach to investigate stability with no delays is to construct a new map that has points of the cycle as equilibriums and then linearize about the equilibrims. Our approach is to construct an auxiliary system that has the cycle elements as coordinates of the equilibrium vector. So, instead of studying cycles of a scalar system we study the equilibrium of a vector system.

In vector case the standard approach produces a characteristic equation as a result of applying the chain rule to the new system, while our approach produces the same equation as a consequence of the non-degenerate property for the auxiliary system linearized around equilibrium.

At this level the advantages of our approach are not visible, but later on they will be more transparent.

For a system with time delays the standard approach leads to a system with dimension that increases with delays. The characteristic polynomials of such systems appear in a standard form that makes impossible analysis on Schur stability, thus useless. Our approach leads to a very well structured system where the characteristic polynomial appears factorized with each factor in a special form that enables stability analysis. Moreover, this new form (see (22) on p. 113) is very compact and is convenient in applications.

2. Main results. A. Standard approach. Let us consider the scalar case of the system (1). Based on a cycle $\left\{\eta_{1}, \ldots, \eta_{T}\right\}$ let us construct a new dynamical system

$$
x_{k+T}=f^{(T)}\left(x_{k}\right),
$$

which iterates $T$ times the initial map. Therefore

$$
f^{(T)}(x)=f\left(f^{(T-1)}(x)\right), \ldots, f^{(1)}(x)=f(x) .
$$

Now, let $F(x)=f^{(T)}(x)$ (see the figure below) and denote $x_{0}=y_{0}, x_{T}=y_{1}, \ldots, x_{k T}=$ $y_{k}, \ldots$. Then the system (3) has changed to

$$
y_{k+1}=F\left(y_{k}\right)
$$

In fact for any $j=0, \ldots, T-1$ we can write

$$
x_{j}=y_{0}, \quad x_{j+T}=y_{1}, \ldots, x_{j+k T}=y_{k}, \ldots
$$

and obtain the same dynamical system (4).

Note that the system (4) has $T$ equilibriums $\eta_{1}, \ldots, \eta_{T}$. Then the cycle $\left\{\eta_{1}, \ldots, \eta_{T}\right\}$ of the system (1) is asymptotically locally stable if and only if all equilibriums $\eta_{1}, \ldots, \eta_{T}$ of the system (4) are asymptotically locally stable. 


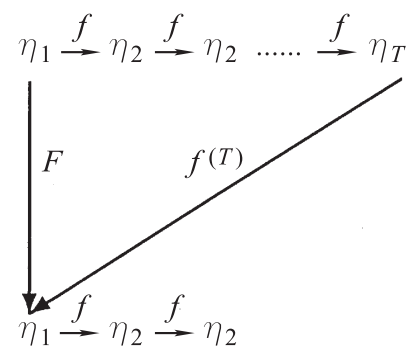

Definition of $F$ map

The multiplier of every equilibrium $\eta_{j}$ of the system (4) is $F^{\prime}\left(\eta_{j}\right), j=1, \ldots, T$. By the chain rule

$$
F^{\prime}\left(\eta_{1}\right)=f^{\prime}\left(f\left(f \ldots f\left(\eta_{1}\right)\right)\right) \cdot f^{\prime}\left(f \ldots f\left(\eta_{1}\right)\right) \cdots f^{\prime}\left(\eta_{1}\right)=\mu_{T} \cdots \mu_{1}
$$

where $\mu_{j}=f^{\prime}\left(\eta_{j}\right)$. It is clear that $F^{\prime}\left(\eta_{j}\right)=\mu_{T} \cdots \mu_{1}, j=1, . ., T$. The quantity $\mu_{T} \cdots \mu_{1}$ is called a multiplier for the cycle $\left\{\eta_{1}, \ldots, \eta_{T}\right\}$. The condition of the local asymptotic stability is $\left|\mu_{T} \cdots \mu_{1}\right|<1$.

B. Alternative approach. We suggest considering a system

$$
\left\{\begin{array}{rll}
x_{k+1} & =f\left(x_{k}\right) \\
x_{k+2} & =f\left(x_{k+1}\right) \\
\cdots \cdots & \cdots & \cdots \cdots \cdots \cdots \cdots \\
x_{k+T} & = & f\left(x_{k+T-1}\right)
\end{array}\right.
$$

Let

$z_{1}=\left(\begin{array}{c}x_{1} \\ x_{2} \\ \vdots \\ x_{T}\end{array}\right), \quad z_{2}=\left(\begin{array}{c}x_{T+1} \\ x_{T+2} \\ \vdots \\ x_{2 T}\end{array}\right), \cdots, z_{k}=\left(\begin{array}{c}x_{(k-1) T+1} \\ x_{(k-2) T+2} \\ \vdots \\ x_{k T}\end{array}\right), z_{k+1}=\left(\begin{array}{c}x_{k T+1} \\ x_{k T+2} \\ \vdots \\ x_{(k+1) T}\end{array}\right), \ldots$

A very simple but a very important observation is that (5) can be written in the following form:

$$
z_{k+1}=\left(\begin{array}{c}
f\left(x_{k T}\right) \\
f\left(x_{k T+1}\right) \\
\vdots \\
f\left(x_{k T+T-1}\right)
\end{array}\right)
$$

Let us note that (6) is a dynamical system $z_{k+1}=\tilde{F}\left(z_{k}\right)$ where

$$
\tilde{F}\left(z_{k}\right)=\left(\begin{array}{c}
f^{(1)}\left(x_{k T}\right) \\
f^{(2)}\left(x_{k T}\right) \\
\vdots \\
f^{(T)}\left(x_{k T}\right)
\end{array}\right) .
$$

Our novelty is that the asymptotically local stability of the cycle $\left\{\eta_{1}, \ldots, \eta_{T}\right\}$ of the system (1) corresponds the asymptotically local stability of the equilibrium 


$$
\left(\begin{array}{c}
\eta_{1} \\
\vdots \\
\eta_{T}
\end{array}\right)
$$

of the system (5).

In the standard approach the cycle corresponds to $T$ equilibriums of the scalar dynamical systems. In our approach this cycle corresponds to one equilibrium of the vector system. So far these two approaches seems absolutely equivalent. The difference will be visible when we start to consider the systems with time delays, especially when the number of the used delays is much larger compared to the size of the cycle.

To compute multipliers we have to linearize the system (5) in the neighborhood of the equilibrium (8)

$$
\left(\begin{array}{c}
\delta_{k}^{(1)} \\
\vdots \\
\delta_{k}^{(T)}
\end{array}\right)=z_{k}-\left(\begin{array}{c}
\eta_{1} \\
\vdots \\
\eta_{T}
\end{array}\right)
$$

and extract the linear part. One can linearize either system (6) or system (7). It turns out that it is more convenient to linearize system (6) because in that case we can avoid dealing with a superposition of the maps. The linearized system has the form

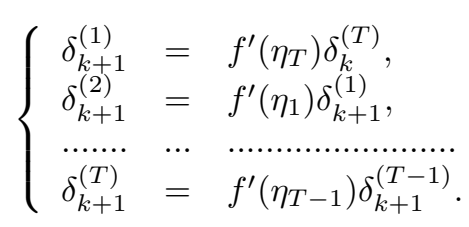

Letting $\delta_{k}^{(j)}=\lambda^{k} s_{j}\left(s_{j} \neq 0\right)$ we obtain

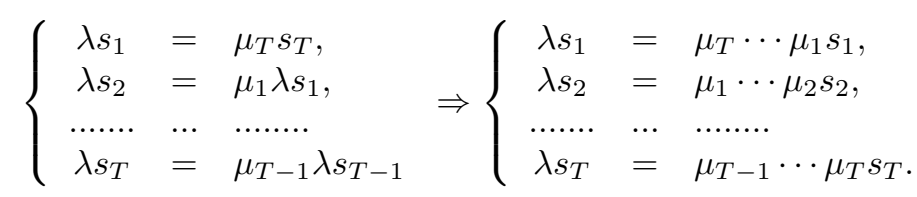

Thus, we come up with the same representation for the multipliers $\lambda=\mu_{T} \cdots \mu_{1}$.

$C$. Vector case. It is amazing that there is no major changes in the vector case.

Namely, in standard approach the Jacobi matrices $M_{j}=f^{\prime}\left(\eta_{j}\right)$ came up and the multipliers are the roots of the characteristic polynomial

$$
\left|\lambda I-M_{T} \cdots M_{1}\right|=0,
$$

while in our case

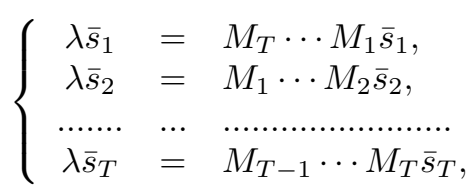

where $\bar{s}_{j}$ are non-zero vectors. Since the eigenvalues of the product of square matrices $A B$ and $B A$ are the same (c. f. $[4$, Ex. 9, p. 55]) we come out with the characteristic equation

$$
\left|\lambda I-M_{T} \cdots M_{1}\right|=0 .
$$


In this case the eigenvalues of the matrix $M_{T} \cdots M_{1}$ are called multipliers of the cycle. If all multipliers in absolute values are less then one then the cycle is asymptotically stable.

D. Systems with time delay. Let us consider a system with time delay in a general form

$$
x_{k+1}=f\left(x_{k}, x_{k-1}, \ldots, x_{k-\tau}\right), \quad f \in \mathbb{R}^{m}, \quad \tau \in \mathbb{Z}_{+} \cdot
$$

And let us study a local stability of the cycle $\left\{\eta_{1}, \ldots, \eta_{T}\right\}$ where $\eta_{j} \in \mathbb{R}^{m}$. In other words for all $k \geqslant \tau+1$ the following equations are valid:

$$
\eta_{(k+1)} \bmod T=f\left(\eta_{k} \bmod T, \eta_{(k-1)} \bmod T, \ldots, \eta_{(k-\tau)} \bmod T\right) .
$$

There abusing notation we assume that $T \bmod T=T$.

Standard. An auxiliary system with respect to the vector of size $m(\tau+1)$ is

$$
z_{k}=\left(\begin{array}{c}
z_{k}^{(1)} \\
z_{k}^{(2)} \\
\vdots \\
z_{k}^{(\tau+1)}
\end{array}\right)=\left(\begin{array}{c}
x_{k-\tau} \\
x_{k-\tau+1} \\
\vdots \\
x_{k}
\end{array}\right)
$$

Then

$$
z_{k+1}=\left(\begin{array}{c}
z_{k+1}^{(1)} \\
z_{k+1}^{(2)} \\
\vdots \\
z_{k+1}^{(\tau+1)}
\end{array}\right)=\left(\begin{array}{c}
z_{k}^{(2)} \\
z_{k}^{(3)} \\
\vdots \\
f\left(z_{k}^{(\tau+1)}, z_{k}^{(\tau)}, \ldots, z_{k}^{(1)}\right)
\end{array}\right)
$$

i. e.

$$
z_{k+1}=F\left(z_{k}\right)
$$

Note, that $F \in \mathbb{R}^{m(\tau+1)}$ in the above formula and is different from the one on the page 2 .

Further, let $z_{k+T}=F\left(\ldots F\left(z_{k}\right)\right)$ be the $T$-times iterated map $F$. This map can be written as a system

$$
y_{k+1}=\Phi\left(y_{k}\right) \text {. }
$$

Let us periodically repeat the elements of the cycle: $\left\{\eta_{1}, \eta_{2}, \ldots, \eta_{T}, \eta_{1}, \eta_{2}, \ldots, \eta_{T}, \ldots\right\}$. The first $\tau+1$ elements of this sequence form a vector

$$
y_{1}^{*}=\left(\begin{array}{c}
\eta_{1} \\
\eta_{2} \\
\vdots
\end{array}\right)
$$

In the same way we define the vectors

$$
y_{2}^{*}=\left(\begin{array}{c}
\eta_{2} \\
\eta_{3} \\
\vdots
\end{array}\right), \ldots, \quad y_{T}^{*}=\left(\begin{array}{c}
\eta_{T} \\
\eta_{1} \\
\vdots
\end{array}\right)
$$

It is clear that the vectors $y_{1}^{*}, \ldots, y_{T}^{*}$ are equilibriums of the system (10).

Then the cycle $\left\{\eta_{1}, \ldots, \eta_{T}\right\}$ of the system (9) is asymptotically locally stable if and only if all equilibriums $y_{1}^{*}, \ldots, y_{T}^{*}$ of the system (10) are asymptotically locally stable. 
For the equilibrium of the system $y_{1}^{*}$ of the system (10) the Jacobi matrix is defined by the formula with $T$ factors

$$
\Phi^{\prime}\left(y_{1}^{*}\right)=F^{\prime}\left(y_{T}^{(*)}\right) \cdots F^{\prime}\left(y_{1}^{(*)}\right),
$$

where the matrix $F^{\prime}\left(y_{j}^{*}\right)$ has dimensions $m(\tau+1) \times m(\tau+1)$ and is equal to

$$
F^{\prime}\left(y_{j}^{*}\right)=\left(\begin{array}{ccccc}
O & I & O & \ldots & O \\
O & O & I & \ldots & O \\
\ldots & & & & \\
O & O & O & \ldots & I \\
Q_{1}^{(j)} & Q_{2}^{(j)} & Q_{3}^{(j)} & \ldots & Q_{\tau+1}^{(j)}
\end{array}\right) .
$$

There the matrices $O$ and $I$ are zero and unit matrices correspondingly and are of the dimensions $m \times m$. Further,

$$
Q_{r}^{(j)}=\left.\frac{\partial f}{\partial z^{(r)}}\right|_{y_{j}^{*}}, \quad r=1, \ldots, \tau+1, \quad j=1, \ldots, T,
$$

i. e. the value of the derivative evaluated at the point $y_{j}^{*}$.

For all other equilibriums $y_{j}^{*}$ the Jacobi matrices $F^{\prime}\left(y_{j}^{*}\right)$ can be computed in the same manner and

$$
\Phi^{\prime}\left(y_{j}^{*}\right)=F^{\prime}\left(y_{(T+j)}^{(*)} \bmod T\right) \cdots F^{\prime}\left(y_{j}^{(*)}\right),
$$

which can be obtained from (11) by a cyclic permutation of the factors, and therefore the eigenvalues of $\Phi^{\prime}\left(y_{j}^{*}\right)$ coincide for all $j=1, \ldots, T$ (c. f. [4]).

If all eigenvalues of the matrix $\Phi^{\prime}\left(y_{j}^{*}\right)$ which are roots of the polynomial

$$
p_{1}(\lambda)=\left|\lambda I-\Phi^{\prime}\left(y_{j}^{*}\right)\right|
$$

are less then one in absolute values then the cycle of the system (9) locally assymptotical stable.

Note that in the scalar case $m=1$ the matrices $F^{\prime}\left(y_{j}\right)$ are in the Frobenius form. Therefore, the matrix (12) is a generalized form of companion matrix. If system is of special case below, then the product is manageable and the characteristic equation was found in scalar case by means of induction in [1].

Our approach. The system (9) generates the system

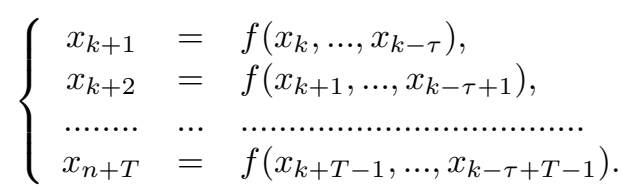

Note that (14) is a dynamical system, which might be seeing by replacing in the right hand side each of $x_{k+1}, \ldots, x_{k+T-1}$ by the functions of $x_{k-\tau}, \ldots, x_{k}$.

If

$$
z_{k}=\left(\begin{array}{c}
z_{k}^{(1)} \\
z_{k}^{(2)} \\
\vdots \\
z_{k}^{(T)}
\end{array}\right)=\left(\begin{array}{c}
x_{(k-1) T+1} \\
x_{(k-1) T+2} \\
\vdots \\
x_{k T}
\end{array}\right), \quad z_{k} \in \mathbb{R}^{T m},
$$


then

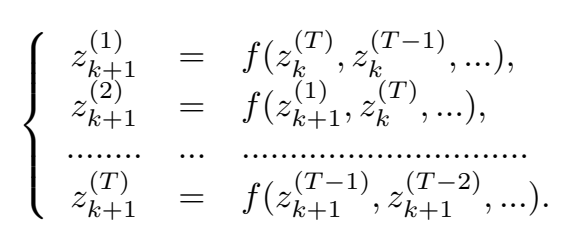

The system (15) can be written in the form

$$
z_{k+1}=\tilde{\Phi}\left(z_{k}, z_{k-1}, \ldots\right), \quad \tilde{\Phi} \in \mathbb{R}^{m T} .
$$

The system (16) has an equilibrium

$$
\left(\begin{array}{c}
\eta_{1} \\
\vdots \\
\eta_{T}
\end{array}\right), \quad \eta_{j} \in \mathbb{R}^{m}, j=1, \ldots, T .
$$

To study the behavior of the solutions to the system (16) in the neighborhood of the equilibrium (17) let us make a substitution

$$
\delta_{k}^{(j)}=z_{k}^{(j)}-\eta_{j}, \quad k=0,1, \ldots, \quad j=1, \ldots, T .
$$

So, instead of linearizing of the system (16) one can linearize the equivalent system (15).

As a result we get

$$
\delta_{k+1}=A_{0} \delta_{k+1}+A_{1} \delta_{k}+\ldots
$$

where

$$
\delta_{k}=\left(\begin{array}{c}
\delta_{k}^{(1)} \\
\vdots \\
\delta_{k}^{(T)}
\end{array}\right)
$$

and $A_{j}$ are square matrices of the dimension $m T \times m T$. The matrix $A_{0}$ on the main diagonal and above have zero entries. That means that matrix $E-A_{0}$ is non-degenerate, therefore the system (18) is a dynamical system. Its characteristic equation is

$$
\left|\lambda I-A_{0} \lambda-A_{1}-\ldots\right|=0 .
$$

If all roots of this equation in absolute values are less then one, then the cycle of the system (14) is locally asymptotically stable.

The polynomial $\lambda^{m(\tau+1)-1}\left|\lambda I-A_{0} \lambda-A_{1}-\ldots\right|$ has the same degree, same leading coefficient and the same roots as polynomial (13) therefore coinside with (13).

E. Example. Let us consider a system (9) written in a coordinate form for $\left(x_{k}, y_{k}\right)^{\mathrm{T}} \in$ $\mathbb{R}^{2}, k=0,1, \ldots$, where $\mathrm{T}$ means transposition

$$
\left\{\begin{array}{l}
x_{k+1}=f\left(x_{k}, y_{k}, x_{k-1}, y_{k-1}, x_{k-2}, y_{k-2}\right) \\
y_{k+1}=g\left(x_{k}, y_{k}, x_{k-1}, y_{k-1}, x_{k-2}, y_{k-2}\right)
\end{array}\right.
$$

Let us assume that the system (19) has a cycle $\bar{\eta}_{1}, \bar{\eta}_{2}$ of the length 2

$$
\bar{\eta}_{1}=\left(\begin{array}{c}
\eta_{11} \\
\eta_{12}
\end{array}\right), \quad \bar{\eta}_{2}=\left(\begin{array}{c}
\eta_{21} \\
\eta_{22}
\end{array}\right),
$$


i. e.

$$
\begin{aligned}
& \left\{\begin{array}{l}
\eta_{21}=f\left(\eta_{11}, \eta_{12}, \eta_{21}, \eta_{22}, \eta_{11}, \eta_{12}\right), \\
\eta_{22}=g\left(\eta_{11}, \eta_{12}, \eta_{21}, \eta_{22}, \eta_{11}, \eta_{12}\right),
\end{array}\right. \\
& \left\{\begin{array}{l}
\eta_{11}=f\left(\eta_{21}, \eta_{22}, \eta_{11}, \eta_{12}, \eta_{21}, \eta_{22}\right), \\
\eta_{12}=g\left(\eta_{21}, \eta_{22}, \eta_{11}, \eta_{12}, \eta_{21}, \eta_{22}\right),
\end{array}\right.
\end{aligned}
$$

and $\bar{\eta}_{1} \neq \bar{\eta}_{2}$.

Thus, for the system (19) we have $m=2, \tau=2$ and $T=2$. We came up with the following problem: write the characteristic equation for checking the local asymptotic stability of the cycle (20).

Standard approach. Define the vector of the size $m(\tau+1)=6$

$$
z_{k}=\left(\begin{array}{c}
z_{k}^{(1)} \\
z_{k}^{(2)} \\
z_{k}^{(3)}
\end{array}\right)
$$

where

$$
z_{k}^{(1)}=\left(\begin{array}{c}
x_{k-2} \\
y_{k-2}
\end{array}\right), \quad z_{k}^{(2)}=\left(\begin{array}{c}
x_{k-1} \\
y_{k-1}
\end{array}\right), \quad z_{k}^{(3)}=\left(\begin{array}{c}
x_{k} \\
y_{k}
\end{array}\right) .
$$

Then the system (19) is equivalent to the system

$$
\left[\begin{array}{c}
z_{k+1}^{(1)} \\
z_{k+1}^{(2)} \\
z_{k+1}^{(3)}
\end{array}\right]=\left[\begin{array}{c}
z_{k}^{(2)} \\
z_{k}^{(3)} \\
f\left(x_{k}, y_{k}, x_{k-1}, y_{k-1}, x_{k-2}, y_{k-2}\right) \\
g\left(x_{k}, y_{k}, x_{k-1}, y_{k-1}, x_{k-2}, y_{k-2}\right)
\end{array}\right]
$$

This system define the map

$$
\left[\begin{array}{l}
\xi_{1} \\
\xi_{2} \\
\xi_{3} \\
\xi_{4} \\
\xi_{5} \\
\xi_{6}
\end{array}\right] \rightarrow\left[\begin{array}{c}
\xi_{3} \\
\xi_{4} \\
\xi_{5} \\
\xi_{6} \\
f\left(\xi_{5}, \xi_{6}, \xi_{3}, \xi_{4}, \xi_{1}, \xi_{2}\right) \\
g\left(\xi_{5}, \xi_{6}, \xi_{3}, \xi_{4}, \xi_{1}, \xi_{2}\right)
\end{array}\right]
$$

which Jacobi matrix evaluated at the points

$$
y_{1}^{*}=\left(\begin{array}{c}
\eta_{11} \\
\eta_{12} \\
\eta_{21} \\
\eta_{22} \\
\eta_{11} \\
\eta_{12}
\end{array}\right) \quad \text { and } \quad y_{2}^{*}=\left(\begin{array}{c}
\eta_{21} \\
\eta_{22} \\
\eta_{11} \\
\eta_{12} \\
\eta_{21} \\
\eta_{22}
\end{array}\right)
$$

correspondingly are

$$
F^{\prime}\left(y_{\sigma}^{*}\right)=\left(\begin{array}{cccccc}
0 & 0 & 1 & 0 & 0 & 0 \\
0 & 0 & 0 & 1 & 0 & 0 \\
0 & 0 & 0 & 0 & 1 & 0 \\
0 & 0 & 0 & 0 & 0 & 1 \\
\phi_{\sigma 1} & \phi_{\sigma 2} & \phi_{\sigma 3} & \phi_{\sigma 4} & \phi_{\sigma 5} & \phi_{\sigma 6} \\
\gamma_{\sigma 1} & \gamma_{\sigma 2} & \gamma_{\sigma 3} & \gamma_{\sigma 5} & \gamma_{\sigma 6} & \gamma_{\sigma 2}
\end{array}\right), \quad \sigma=1,2
$$


Above

$$
\left.\frac{\partial f}{\partial \xi_{j}}\right|_{y_{1}^{*}}=\phi_{1, j},\left.\frac{\partial g}{\partial \xi_{j}}\right|_{y_{1}^{*}}=\gamma_{1, j},\left.\frac{\partial f}{\partial \xi_{j}}\right|_{y_{2}^{*}}=\phi_{2, j},\left.\frac{\partial g}{\partial \xi_{j}}\right|_{y_{2}^{*}}=\gamma_{2, j}, \quad j=1, \ldots, 6 .
$$

Then

$$
\Phi^{\prime}\left(y_{1}^{*}\right)=F^{\prime}\left(y_{1}^{*}\right) F^{\prime}\left(y_{2}^{*}\right) .
$$

The characteristic polynomial is

$$
p_{1}(\lambda):=\left|\lambda I-F^{\prime}\left(y_{1}^{*}\right) F^{\prime}\left(y_{2}^{*}\right)\right|,
$$

where $I$ is a unit matrix of the dimensions $6 \times 6$.

Alternative approach. The system (14) has a form

$$
\left\{\begin{array}{l}
x_{k+1}=f\left(x_{k}, y_{k}, x_{k-1}, y_{k-1}, x_{k-2}, y_{k-2}\right) \\
y_{k+1}=g\left(x_{k}, y_{k}, x_{k-1}, y_{k-1}, x_{k-2}, y_{k-2}\right) \\
x_{k+2}=f\left(x_{k+1}, y_{k+1}, x_{k}, y_{k}, x_{k-1}, y_{k-1}\right) \\
y_{k+2}=g\left(x_{k+1}, y_{k+1}, x_{k}, y_{k}, x_{k-1}, y_{k-1}\right)
\end{array}\right.
$$

Define a vector

$$
z_{k}=\left(\begin{array}{c}
z_{k}^{(1)} \\
z_{k}^{(2)}
\end{array}\right)
$$

where

$$
z_{k}^{(1)}=\left(\begin{array}{c}
x_{2(k-1)+1} \\
y_{2(k-1)+1}
\end{array}\right), \quad z_{k}^{(2)}=\left(\begin{array}{c}
x_{2 k} \\
y_{2 k}
\end{array}\right)
$$

then

$$
z_{k+1}=\left(\begin{array}{c}
x_{2 k+1} \\
y_{2 k+1} \\
x_{2 k+2} \\
y_{2 k+2}
\end{array}\right)=\left(\begin{array}{c}
f\left(x_{2 k}, y_{2 k}, x_{2 k-1}, y_{2 k-1}, x_{2 k-2}, y_{2 k-2}\right) \\
g\left(x_{2 k}, y_{2 k}, x_{2 k-1}, y_{2 k-1}, x_{2 k-2}, y_{2 k-2}\right) \\
f\left(x_{2 k+1}, y_{2 k+1}, x_{2 k}, y_{2 k}, x_{2 k-1}, y_{2 k-1}\right) \\
g\left(x_{2 k+1}, y_{2 k+1}, x_{2 k}, y_{2 k}, x_{2 k-1}, y_{2 k-1}\right)
\end{array}\right)
$$

After substituting in the third and the forth equations the values for $x_{2 k+1}$ and $y_{2 k+1}$ by their values from the first two equations we get a system

$$
z_{k+1}=\tilde{\Phi}\left(z_{k}, z_{k-1}\right)
$$

Making a substitution $\delta_{k}^{(j)}=\bar{\eta}_{j}-z_{k}^{(j)}, j=1,2$, we linearize the system. Then (18) takes the form

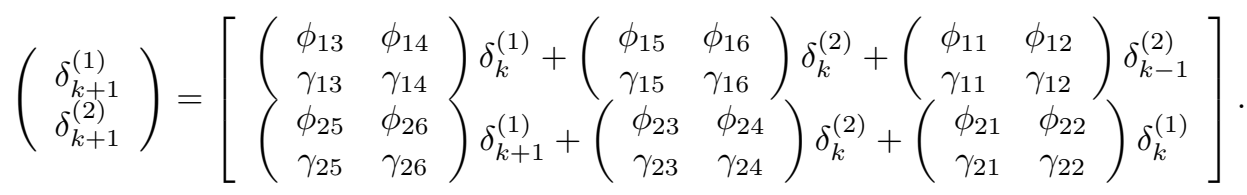

From there we get the characteristic polynomial

$$
p_{2}(\lambda):=\mid \lambda^{2} I-\left(\begin{array}{cccc}
0 & 0 & 0 & 0 \\
0 & 0 & 0 & 0 \\
\phi_{25} & \phi_{26} & 0 & 0 \\
\gamma_{25} & \gamma_{26} & 0 & 0
\end{array}\right) \lambda^{2}-
$$




$$
-\left(\begin{array}{cccc}
\phi_{13} & \phi_{14} & \phi_{15} & \phi_{16} \\
\gamma_{13} & \gamma_{14} & \gamma_{15} & \gamma_{16} \\
\phi_{21} & \phi_{22} & \phi_{23} & \phi_{24} \\
\gamma_{21} & \gamma_{22} & \gamma_{23} & \gamma_{24}
\end{array}\right) \lambda-\left(\begin{array}{cccc}
0 & 0 & \phi_{11} & \phi_{12} \\
0 & 0 & \gamma_{11} & \gamma_{12} \\
0 & 0 & 0 & 0 \\
0 & 0 & 0 & 0
\end{array}\right) \mid
$$

One can verify that $p_{1}(\lambda)$ and $\lambda^{-2} p_{2}(\lambda)$ are same.

3. Special case. Our approach in contrast to the standard one is allowed for the systems of the special type (2) which is

$$
x_{k+1}=a_{1} f\left(x_{k}\right)+a_{2} f\left(x_{k-T}\right)+\ldots+a_{N} f\left(x_{k-(N-1) T}\right),
$$

immediately write the characteristic polynomial. Then

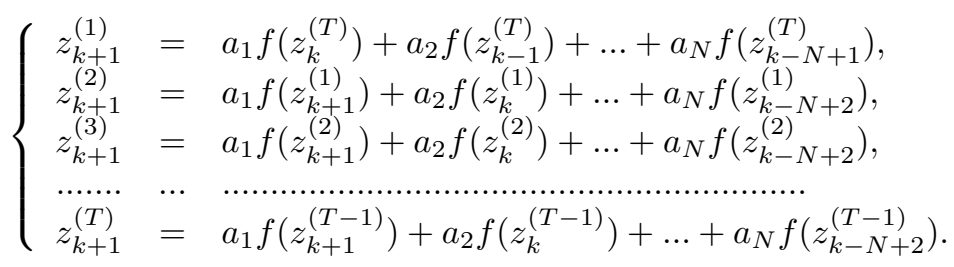

Let us denote Jacobi matrices $f^{\prime}\left(\eta_{j}\right)$ of the dimension $m \times m$ by $M_{j}, j=1, \ldots, T$. Let $z_{k}^{(j)}=\eta_{j}+\delta_{k}^{(j)}$. By linearizing we get

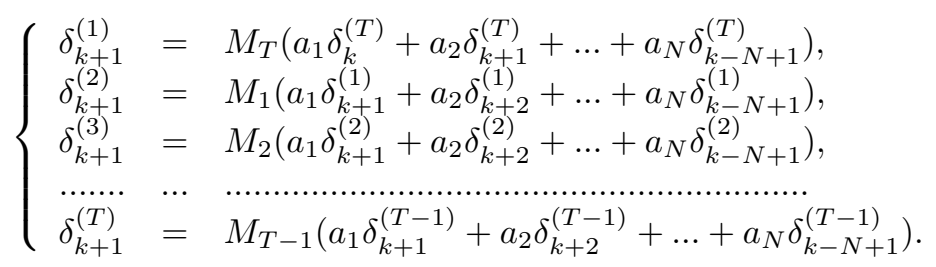

Letting $\delta_{k}^{(j)}=\lambda^{k} \bar{s}_{j}$ we obtain $\left(\bar{s}_{j} \neq 0\right)$

$$
\left\{\begin{array}{ccc}
\lambda^{k+1} \bar{s}_{1} & =M_{T}\left(a_{1} \lambda^{k}+\ldots+a_{N} \lambda^{k-N+1}\right) \bar{s}_{T}, \\
\lambda^{k+1} \bar{s}_{2} & =M_{1}\left(a_{1} \lambda^{k+1}+\ldots+a_{N} \lambda^{k-N+2}\right) \bar{s}_{1} \\
\ldots \ldots \ldots & \ldots & \ldots \ldots \ldots \ldots \ldots \ldots \ldots \ldots \ldots \ldots \ldots \ldots \ldots \ldots \ldots \ldots \ldots \ldots \ldots \ldots \ldots \ldots \ldots \ldots \ldots \ldots \ldots \ldots \ldots \ldots \ldots \ldots \ldots \\
\lambda^{k+1} \bar{s}_{T} & = & M_{T-1}\left(a_{1} \lambda^{k+1}+\ldots+a_{N} \lambda^{k-N+2}\right) \bar{s}_{T-1} .
\end{array}\right.
$$

Denote $p(\lambda)=a_{1} \lambda^{N}+\ldots+a_{N}$. Then

$$
\left\{\begin{array}{ccc}
\lambda^{N} \bar{s}_{1} & = & M_{T} p(\lambda) \bar{s}_{T} \\
\lambda^{N-1} \bar{s}_{2} & = & M_{1} p(\lambda) \bar{s}_{1} \\
\ldots \ldots & \cdots & \cdots \ldots \ldots \ldots \ldots \ldots \ldots \\
\lambda^{N-1} \bar{s}_{T} & = & M_{T-1} p(\lambda) \bar{s}_{T-1}
\end{array}\right.
$$

Since the eigenvalues of the matrix $M_{T} \cdots M_{1}$ are independent on the cyclic reordering of the product matricies [4], we have

$$
\left|\lambda^{1+(N-1) T} I-M_{T} M_{T-1} \cdots M_{1}(p(\lambda))^{T}\right|=0 .
$$

Let the eigenvalues of the matrix $M_{T} M_{T-1} \cdots M_{1}$ be $\mu_{1}, \ldots, \mu_{m}$. Then changing to the product to the Jordan canonical form one get from (21)

$$
\prod_{j=1}^{m}\left(\lambda^{1+(N-1) T}-\mu_{j}(p(\lambda))^{T}\right)=0 .
$$


In special case and $m=1$ the equivalence of the characteristic polynomials obtain by the standard and by our approach was established in [1].

4. Conclusion. Assume that the system

$$
x_{k+1}=f\left(x_{k}\right), \quad f: A \rightarrow A, A \subset \mathbb{R}^{m},
$$

has an unstable $T$-cycle $\left(\eta_{1}, \ldots, \eta_{T}\right)$. The cycle multipliers $\mu_{1}, \ldots, \mu_{m}$ are zeros of the characteristic polynomial

$$
\operatorname{det}\left(\mu I-\prod_{j=1}^{T} f^{\prime}\left(\eta_{j}\right)\right)=0 .
$$

Assume that the multipliers are known only approximately, i. e. located in a region $M \subset \mathbb{C}$.

Now, let us close the system by a control

$$
u_{n}=-\sum_{j=1}^{N-1} \varepsilon_{j}\left(f\left(x_{n-j T+T}\right)-f\left(x_{n-j T}\right)\right),\left|\varepsilon_{j}\right|<1, j=1, \ldots, N-1 .
$$

The closed-loop system $x_{n+1}=f\left(x_{n}\right)+u_{n}$ can be written as

$$
x_{n+1}=\sum_{k=1}^{N} a_{k} f\left(x_{n-k T+T}\right), \quad \sum_{k=1}^{N} a_{k}=1,
$$

where $a_{k}$ and $\varepsilon_{k}$ are in bijection

$$
\varepsilon_{j}=\sum_{k=j+1}^{N} a_{k}, j=1, \ldots, N-1
$$

Note that the $T$-cycles of the systems (23) and (25) coincide.

The characteristic equation of the linearized around the cycle system is

$$
\prod_{j=1}^{m}\left[\lambda^{T(N-1)+1}-\mu_{j}\left(\sum_{k=1}^{N} a_{k} \lambda^{N-k}\right)^{T}\right]=0, \quad \mu_{j} \in M, j=1, \ldots, m .
$$

It is required to choose the gain $\varepsilon_{j}$ in the control (24) such that

i) $T$-cycle of the system (25) to be locally stable;

ii) the depth of the prehistory $T(N-1)$ in the control $(24)$ to be minimal.

Thanks to formula (22) the above problem can be treated by the use of geometric function theory of complex variables. This theory allows to find necessary metric properties of the exceptional sets of the polynomial mappings of the unit disc

$$
F: \mathbb{D} \rightarrow \mathbb{C}, F(z)=z\left(\sum_{j=1}^{N} a_{j} z^{j-1}\right)^{T}
$$

From there one can find the minimal values of $N$ and the optimal coefficients. The scalar case of $T=1,2$ was treated in $[5,6]$. 
The author would like to express her deep gratitude to Dmitriy Dmitrishin and Paul Hagelstein for the guidance through the problem, useful discussions and for the help in preparation of manuscript.

\section{References}

1. Dmitrishin D., Hagelstein P., Khamitova A., Stokolos A. On the stability of cycles by delayed feedback control. Linear and Multilinear Algebra, 2016, vol. 64, issue 8, pp. 1538-1549.

2. Dmitrishin D., Khamitova A., Stokolos A., Tohaneanu M. Extremal trigonometric polynomials and the problem of optimal stabilization of chaos. Harmonic analysis, partial differential equations, complex analysis, banach spaces, and operator theory. Association for Women in Mathematics Series, 2016, vol. 2 (unpublished).

3. Morgül Ö. On the stability of delayed feedback controllers. Physics Letters A, 2003, vol. 314, pp. $278-285$.

4. Horn R. A., Johnson C. Matrix analysis. Cambridge, Cambridge University Press, 1985, 561 p.

5. Dmitrishin D., Khamitova A. Methods of harmonic analysis in nonlinear dynamics. Comptes rendus mathematique, 2013, vol. 351, issues 9-10, pp. 367-370.

6. Dmitrishin D., Khamitova A., Stokolos A. Fejér polynomials and Chaos. Springer Proceedings in Mathematics and Statistics, 2014, vol. 108, pp. 49-75.

For citation: Khamitova A. D. Characteristic polynomials for a cycle of non-linear discrete systems with time delays. Vestnik of Saint Petersburg University. Series 10. Applied mathematics. Computer science. Control processes, 2016, issue 4, pp. 104-115. DOI: 10.21638/11701/spbu10.2016.410

Статья рекомендована к печати доц. А. П. Жабко.

Статья поступила в редакцию 15 октября 2015 г.

Статья принята к печати 29 сентября 2016 г. 